\title{
SUSTITUCIÓN ESOFÁGICA POR TUBO GÁSTRICO INVERTIDO A RAÍZ DE ESTENOSIS ESOFÁGICA POR INGESTA DE CÁUSTICOS (PRESENTACIÓN DE CASO) HOSPITAL INFANTIL ROBERT REID CABRAL (HIRRC), REPÚBLICA DOMINICANA
}

\section{ESOPHAGEAL REPLACEMENT BY REVERSED GASTRIC TUBE DUE TO ESOPHAGEAL CAUSTIC STENOSIS (CASE PRESENTATION) Robert Reid Cabral Children's Hospital, Dominican Republic}

\author{
Carlos Cabrera Valerio*, Elvyn Alcántara**, Andreína Moreno Reyes***, Ariadna L. García \\ Risk $^{* * * *}$, Melissa L. Quiroz Fernández****
}

Fecha recibido: 22 de agosto 2018 • Fecha aprobado: 6 de noviembre 2019

Cómo citar: Cabrera Valerio C, Alcántara E, Moreno Reyes A, García Risk AL, Quiroz Fernández ML. Sustitución esofágica por tubo gástrico invertido a raíz de estenosis esofágica por ingesta de cáusticos (presentación de caso). Hospital Infantil Robert Reid Cabral (HIRRC) República Dominicana. cysa [Internet]. 11 abr. 2019 [citado 12 abr. 2019];1(1):67-2. Available from: https:// revistas.intec.edu.do/index.php/cisa/article/view/1333

\section{Resumen}

Introducción: La lesión esofágica por cáusticos debida a la ingestión de álcalis o, con menos frecuencia, ácidos, puede ser accidental en niños o por intento de suicidio en adolescentes y adultos. La secuela esofágica de la ingestión de un cáustico es una cicatriz en la pared del esófago que se relaciona con frecuencia con la formación de estenosis intensa y en muchas ocasiones, se requiere de un conducto que restablezca la función del esófago.

Material y método: En el siguiente trabajo se expone el caso clínico de un paciente masculino de 7 años de edad, presentando sialorrea y dificultad para la deglución debido a ingesta de sustancia cáustica de manera accidental. Se realizó endoscopia que reporta estenosis esofágica de $2 \mathrm{~mm}$, para garantizar su alimentación se le practicó una gastrostomía. No fue posible el esquema de dilatación, con la necesidad de proceder a una sustitución esofágica

${ }^{*}$ Cirujano Pediátrico del Hospital Infantil Dr. Robert Reid Cabral (HIRRC)

** Cirujano Pediátrico del Hospital Infantil Dr. Robert Reid Cabral (HIRRC)

***Médico General.

****Médico General

***** Médico General por tubo gástrico invertido vía retroesternal. Presentó una complicación postquirúrgica de neumotórax. Una estancia de 12 días con inicio de la vía oral a los 11 días de postquirúrgico, previa realización de esofagograma.

Conclusión El propósito consiste en describir la presentación y el progreso clínico del paciente luego de esta técnica de sustitución esofágica por tubo gástrico invertido vía retroesternal.

Palabras Claves: Cáusticos; Estenosis Esofágica; Esófago; Sustitución Esofágica; Tubo Gástrico.

\section{Abstract}

Introduction: Caustic esophageal injury due to ingestion of alkalis or, less frequently, acids, may be accidental in children or by attempted suicide in adolescents and adults. The esophageal sequela of the ingestion of a caustic is a scar on the wall of the esophagus that is often related to the formation of severe stenosis and in many cases, it requires a conduit to restore the function of the esophagus.

Carloscabrera84@gmail.com

Enriquedanielalcantaragil2gmail.com

Andreinamorenor3@gmail.com

Ariadanlgr02@gmail.com

Melquiroz95@gmail.com 


\section{Carlos Cabrera Valerio, Elvyn Alcántara, Andreína Moreno Reyes, Ariadna L. García Risk y Melissa L. Quiroz Fernández}

Material and method: In the following work the clinical case of a male patient of 7 years of age is presented, presenting hypersalivation and difficulty in swallowing due to ingestion of caustic substance accidentally, endoscopy was performed reporting esophageal stenosis of $2 \mathrm{~mm}$, to guarantee its feeding was a gastrostomy, the dilatation scheme was not possible, with the need to proceed to an esophageal replacement by inverted gastric tube via retrosternal. He presented a postoperative complication of pneumothorax. A 12 day stay with beginning of the oral route at 11 days post-surgery after performing an esophagogram.

Conclusion: The purpose is to describe the presentation and clinical progress of the patient after this technique of esophageal replacement by inverted gastric tube via retrosternal.

Key Words: Caustics; Esophageal Stenosis; Esophagus; Esophageal Substitution; Gastric Tube.

\section{Introducción}

La lesión esofágica por cáusticos debida a la ingestión de álcalis o, con menos frecuencia, ácidos, puede ser accidental en nińos o por intento de suicidio en adolescentes y adultos. La secuela esofágica de la ingestión de un cáustico es una cicatriz en la pared del esófago que se relaciona con frecuencia con la formación de estenosis intensa y en muchas ocasiones, se requiere la dilatación repetida, lo que genera la necesidad de una reconstrucción de un conducto que restablezca la función del esófago ${ }^{123}$.

Considerando que las enfermedades esofágicas complejas tienen gran impacto en la sobrevida y en la calidad de vida de la población pediátrica, existe un permanente debate en cuanto a las técnicas quirúrgicas más adecuadas para el tratamiento de estos pacientes, dentro de ellas se encuentran la interposición del colon, la interposición gástrica, la sustitución yeyunal y el reemplazo esofágico con tubo gástrico ${ }^{34}$.

"La técnica del tubo gástrico invertido como opción en el reemplazo del esófago, que emplea un tubo creado a partir de la curvatura mayor invertida, fue descrita por Beck y Carrell en 1905. No obstante, a mediados de la década de 1970, el reemplazo del esófago con tubo gástrico se tornó viable en niños. En 1972, se publicaron una serie de casos de 53 pacientes, lo que popularizó la técnica ya que presentaba ventajas tales como la posibilidad de alcanzar la región cervical y faríngea por cualquiera de las rutas mediastinales, anastomosis única, la restauración satisfactoria de la deglución y la creación rápida con una engrapadora. Sin embargo, la larga línea de suturas gástricas y el suministro sanguíneo irregular pueden considerarse como posibles desventajas"1 4

"Estos dispositivos pueden instalarse desde el antro hacia arriba o desde el fondo hacia abajo y pueden construirse de manera que exista suficiente tubo gástrico para llegar al cuello. Los resultados a largo plazo de la interposición de un tubo gástrico son mejores en comparación con la interposición de colon, sin contar que el tubo gástrico es más fácil de construir"13.

\section{Reporte del Caso}

Paciente masculino de 7 años de edad, proveniente de Villa Mella, Santo Domingo, presentando sialorrea y dificultad para la deglución luego de la ingesta de sustancia cáustica (desgrasante) a los 4 años de edad, de forma accidental, que le produjo estenosis esofágica confirmado por esofagograma y endoscopia que reporta estenosis de $2 \mathrm{~mm}$ a $18 \mathrm{~cm}$ de la arcada dentaria por lo cual se realiza gastrostomía para garantizar alimentación. No fue posible el esquema de dilatación esofágica en este paciente por lo que en mayo 2018, se procede a realizar sustitución esofágica por tubo gástrico invertido.

Paciente seguido por el servicio de gastroenterología y nutrición para mantener una adecuada nutrición con los preparados especiales (licuados). Evaluación por el servicio de neumología por los 
procesos respiratorio a repetición, así como evaluación pre quirúrgica por cardiología y anestesiología.

Al examen físico antes de la cirugía, el paciente está en condiciones generales estables con hallazgos positivos de cicatriz post quirúrgica en línea media supraumbilical y gastrostomía en cuadrante superior izquierdo del abdomen.

\section{Figura 1. Esofagograma luego de la ingesta de sustancia cáustica.}

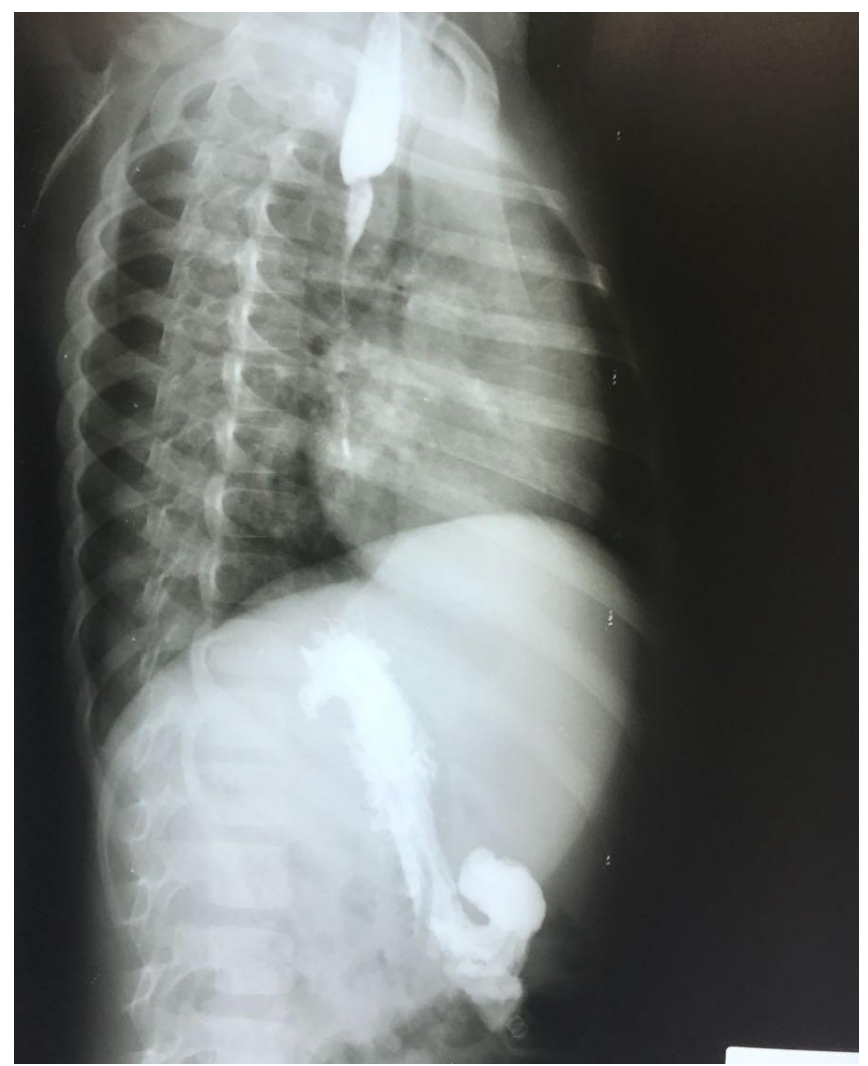

Fuente: Fotos de la clínica de sustitución Esofágica del HIDRRC.

\section{Técnica Quirúrgica}

Previa asepsia y antisepsia, se procede a realizar laparotomía, desmonte de la gastrostomía con cierre gástrico en doble plano, se realiza maniobra de Kocher, luego una piloroplastía, se libera el ligamento gastrocólico de derecha a izquierda, la arcada gastroepiploica se deja unida al estómago y es ligada la arteria gastroepiploica derecha a $2 \mathrm{~cm}$ aproximadamente del píloro, desde este punto se crea el tubo gástrico hacia el fundus gástrico en paralelo a la curvatura mayor utilizando grapadora de anastomosis intestinal lineal tipo GIA con recargas de $60 \mathrm{~mm}$ y $80 \mathrm{~mm}$. Para la ruta hacia el cuello se crea un túnel retroesternal con disección digital, se hace una cervicotomía izquierda, se diseca por planos anatómicos y se secciona el esófago; el segmento distal se cierra en bolsa ciega y el proximal se anastomosa con el tubo gástrico invertido (esofagogastroplastía) ascendido vía retroesternal. Se realiza yeyunostomía por técnica de Stamm para garantizar la alimentación enteral hasta poder iniciar la vía oral. Además, se le coloca una vía central en la vena subclavia derecha para manejo de líquidos y alimentación parenteral.

\section{Figura 2.}

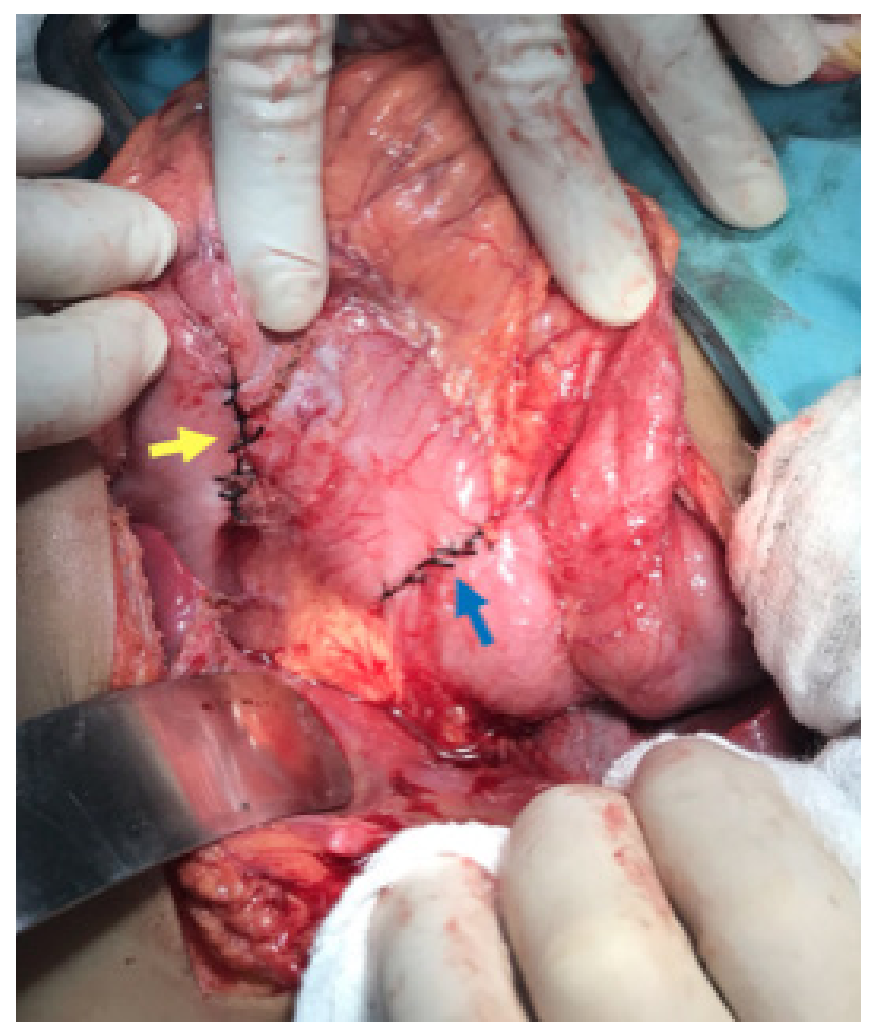

Estómago donde se identifica en la flecha amarilla el cierre de la gastrostomía y en la flecha azul la piloroplastía. Fuente: Fotos de la clínica de sustitución Esofágica del HIDRRC. 


\section{Figura 3}
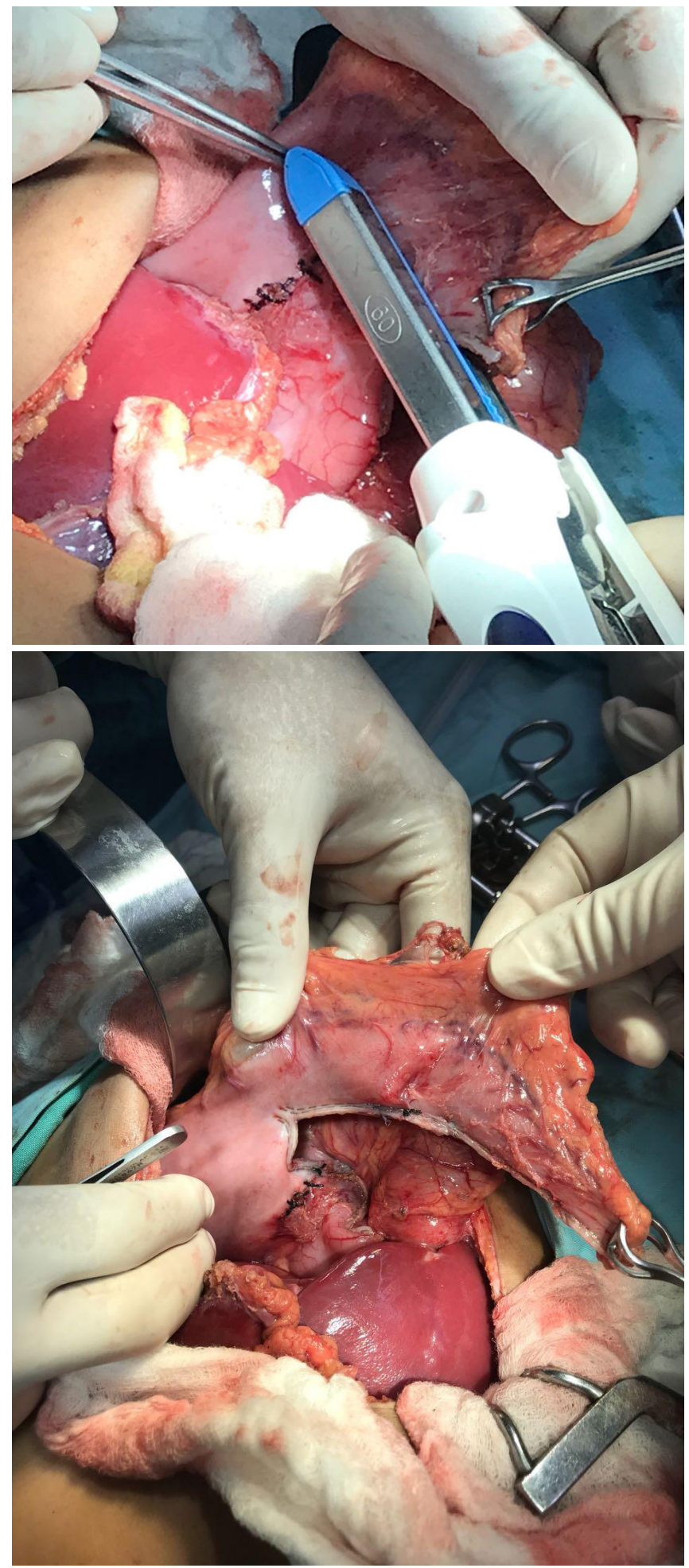

Se puede visualizar la creación del tubo gástrico en la curvatura mayor del estómago. Fuente: Fotos de la clínica de sustitución Esofágica del HIDRRC.
Figura 4

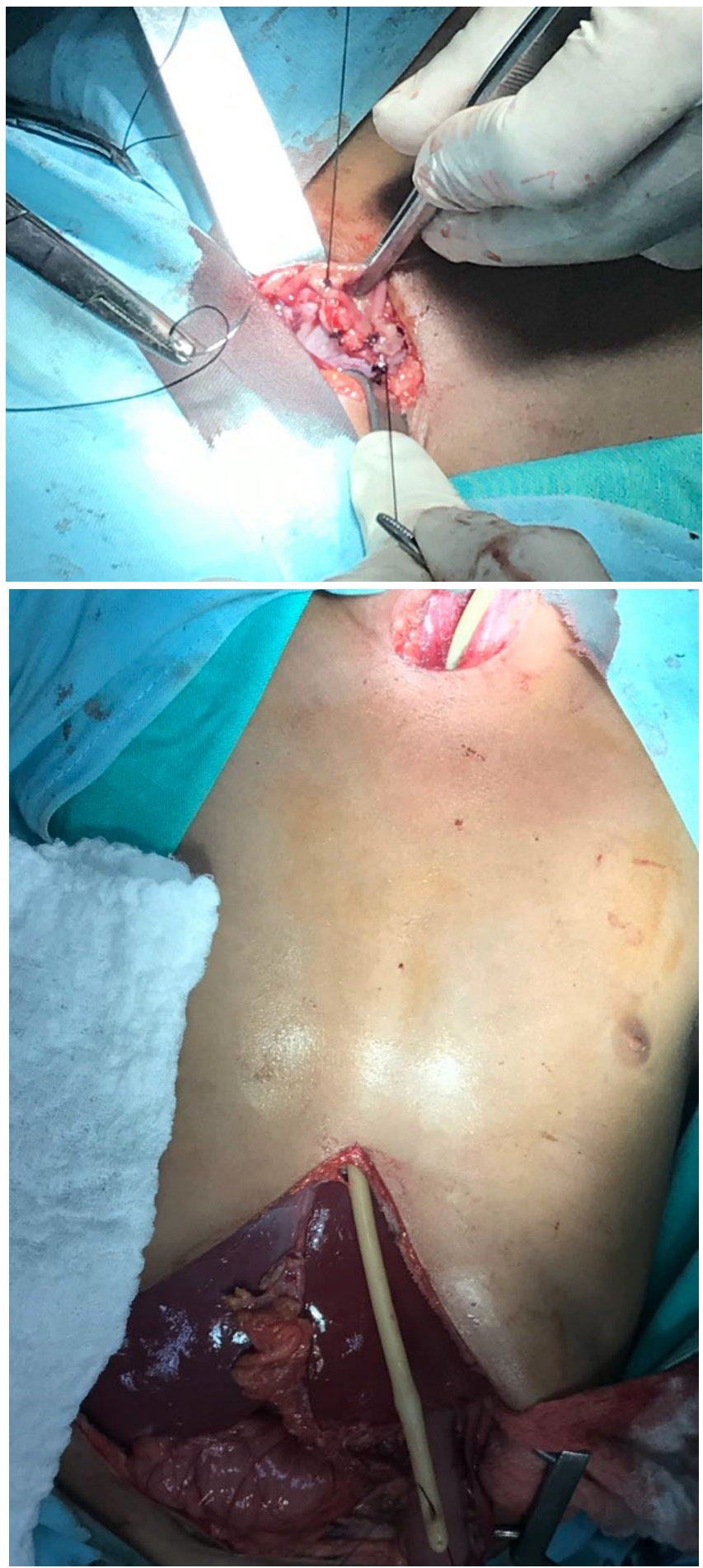

Se asciende tubo gástrico invertido vía retro esternal hasta región cervical izquierda. Luego se procede hacer la esofagogastroplastía cervical.

Fuente: Fotos de la clínica de sustitución Esofágica del HIDRRC 


\section{Discusión}

La ingesta de sustancia cáustica (ISC) continúa siendo una amenaza para la vida en todo el mundo, por lo cual es un problema de salud pública. Las ingestas de productos cáusticos son causa común de lesión esofágica en los niños, por lo que es una problemática de alto costo principalmente en países en desarrollo, como es el nuestro. En los países industrializados el impacto en salud de esta entidad ha disminuido, pero aún sigue siendo la segunda causa de sustitución esofágica en pediatría, a pesar del esfuerzo para la prevención. ${ }^{3456}$

La estenosis esofágica puede ocurrir de 3\% a 57\% de los casos, luego de una ingesta cáustica, y responden a tratamientos médicos y dilataciones en un $64-100 \%$. Si no responden a las dilataciones requieren sustitución esofágica. ${ }^{7}$

Existen diferentes técnicas de reemplazo esofágico sin que haya evidencia de cuál es la mejor técnica en la infancia. Pero el tubo gástrico puede presentar algunas ventajas sobre la técnica convencional de ascenso gástrico, la interposición de colon o el tubo gástrico invertido supercargado, ya que es un procedimiento simple que requiere solamente una anastomosis a nivel cervical. Además, el tubo gástrico invertido facilita un proceso de deglución más fisiológico y permite un mayor aclaramiento esofágico. La elección del injerto depende del cirujano y experiencia. ${ }^{18910}$

Independientemente de la técnica, se han observado complicaciones como la fuga de la anastomosis cervical que es la más frecuente, síntomas respiratorios (neumotórax, neumonía), síntomas gastrointestinales (reflujo gastroesofágico, úlceras del tubo gástrico y hemorragias), así como estenosis de la anastomosis a largo plazo. ${ }^{456111213}$ Nuestro paciente presentó neumotórax derecho como única complicación postquirúrgica. Tuvo una estancia hospitalaria de 12 días con inicio de la vía oral con líquidos claros a los 11 días de postquirúrgico previa realización de esofagograma. Con una evolución en su seguimiento satisfactoria y adecuada deglución de alimentos solidos.

\section{Bibliografía}

1. Ashcraft K, Holcomb G, Murphy J. Pediatric surgery. Philadelphia, Pennsylvania: Elsevier Saunders; 2005.

2. Nelson W, Behrman R, Kliegman R. Nelson essentials of pediatrics. Philadelphia: Saunders; 1998.

3. Herrera-Toro N, Arango-Rave ME, PeñaAguirre L, Silvera-Valera MC.

Resultados de la cirugía de reconstrucción esofágica en pacientes pediátricos con patología esofágica compleja en dos hospitales de alto nivel de Medellín, Colombia, 2006-2016 [Internet] Iatreia. 2017 Oct-Dic;30(4): 369375. DOI 10.17533/udea.iatreia.v30n4a02. Disponible en: https://aprendeenlinea.udea. edu.co/revistas/index.php/iatreia/article/ view/327085

3. Leal Buitrago Carlos, Bernal Santos Felipe, Cabrera Vargas Luis Felipe. Técnica del tubo gástrico invertido como opción para la reconstrucción esofágica, en un hospital de tercer nivel en Bogotá, Colombia: presentación de dos casos y revisión de la literatura. Rev Col Gastroenterol [Internet]. 2017 Mar [cited 2018 June 19] ; 32( 1 ): 47-54. Available from: http://www.scielo.org.co/scielo.php?script=sci_arttext\&pid $=$ S0120-99572017000100007\&lng=en:http: //dx.doi.org/https://doi.org/10.22516/2500 7440.129 .

4. Mendez M., Pérez A., Pérez D P. Quemadura esofágica por ingesta de cáusticos en una población pediátrica Hospital de la MisericordiaBogotá 1996 al 2009. CIRUPED, Vol 2, N2, Junio de 2012. www.ciruped.org 


\section{Carlos Cabrera Valerio, Elvyn Alcántara, Andreína Moreno Reyes, Ariadna L. García Risk y Melissa L. Quiroz Fernández}

5. Elfiky, Mahmoud M.A. et al. Gastric tube esophagoplasty for pediatric esophageal replacement [Internet] Journal of Pediatric Surgery, Volume 52, Issue 4, 657 - 662. Disponible en:https://www.ncbi.nlm.nih.gov/ pubmed/28063680

6. Montalva L, Viala J, Michelet D, Frade F. et al... Total Laparoscopic Approach for Transhiatal Esophagectomy and Gavriliu's Esophagoplasty in Children with Caustic Esophageal Injuries.2017; Vol 27(10):1085 1090. Paris, France.

7. Lee, Hui Qing et al. Long-gap -oesophageal atresia: comparison of delayed primary anastomosis and esophageal replacement with gastric tube [Internet] Journal of Pediatric Surgery, Volume 49 , Issue 12, 1762 1766. https://www.jpedsurg.org/article/ S0022-3468(14)00550-8/pdf

8. Choi, Rosa $S$ et al. Esophageal replacement in children who have caustic pharyngoesophageal strictures [Internet] Pediatric Surgery, Volume 32, Issue 7, $1083-1088$ Disponible en:https://www.ncbi.nlm.nih.gov/ pubmed/9247239

9. Ein, Sigmund H. Gastric tubes in children with caustic esophageal injury: A 32-year review [Internet] Journal of Pediatric Surgery, Volume 33, Issue 9, 1363 - 1365 Disponible en: https://www.jpedsurg.org/article/
S0022-3468(98)90008-2/fulltext? $\operatorname{code}=$ yjpsu-site

10. Torres Naranjo, Yudith Leidys, Trinchet Soler, Rafael M, Hidalgo Marrero, Yanet, Quintero Nicó, Wilmer, Manzano Suárez, Jianeya, Cárdenas Salazar, Hosorio. Sustitución esofágica temprana en un paciente con atresia esofágica de brecha larga [Internet]. 2015 Dic [citado 2018 Jun 19]; 19( 4 ): 798-807. Disponible en: http://scielo. sld.cu/scielo.php?script $=$ sci_arttext \&pi$\mathrm{d}=$ S1560-43812015000400021\&lng=es.

11. Zanotti Cavazonni. Reparación de la atresia de esófago de cabos distantes o lesiones cáusticas con tubo gástrico. Revista de Cir Infantil 7 (4) [Internet]. 1997. Disponible en: http:// www.acacip.org.ar/revista/070404.pdf

12. Aquino, José Luis, Braga de, Said Marcelo, Brandi, Luis Antonio, Oliveira, Jean Marc Vinagre Prado de, Maziero Diana, Merhi Vânia, Leandro. Tubo gástrico isoperistáltico de grande curvatura gástrica com sutura mecânica no tratamento cirúrgico do câncer de esôfago irressecável. ABCD, arq. bras. cir. dig. [Internet]. 2009 Sep [cited 2018 June 19]; 22( 3 ): 147-152. Available from: http://www. scielo.br/scielo.php?script=sci_arttext\&pi$\mathrm{d}=$ S0102-67202009000300003\&lng=en . http://dx.doi.org/10.1590/S0102-672020 09000300003. 